\title{
Revista Psicologia: Organizações e Trabalho
}

Psychology: Organizations and Work Journal

Revista Psicología: Organizaciones y Trabajo

ISSN 1984-6657 • doi: 10.17652/rpot/2019.4.17418

\section{Quanto mais melhor? 0 efeito da compatibilidade pessoa-trabalho no engajamento}

\author{
Juliana Barreiros Porto ${ }^{1, a}$, Lígia Carolina Oliveira-Silva ${ }^{a}$, Elis Calcagno Martins ${ }^{\mathrm{b}}$
}

Universidade de Brasília, Distrito Federal, Brasila; Universidade Federal de Uberlândia, Minas Gerais, Brasil ${ }^{b}$

\section{Palavras-chave:}

Compatibilidade pessoa-

trabalho,

engajamento no trabalho, regressão polinomial

\begin{abstract}
Resumo
As preferências do indivíduo têm papel relevante na relação entre desenho do trabalho e engajamento, mas são pouco exploradas. Este estudo adota um enfoque da psicologia positiva e se propõe a investigar duas hipóteses contraditórias, a primeira advinda da teoria de autodeterminação, que indica que a interação entre a característica do trabalho e as preferências do indivíduo não são relevantes para explicar o grau de engajamento (H1) e a segunda advinda das teorias de fit que afirma que essa interação acrescenta à explicação do fenômeno (H2). Foram testadas as características do desenho do trabalho de conhecimento, autonomia e suporte social. A amostra de 341 trabalhadores respondeu as escalas de Engajamento e Desenho do Trabalho. Foram realizadas análises fatoriais confirmatórias, regressões polinomiais e análises de superfície do padrão de respostas. As características do trabalho explicaram significativamente o nível de engajamento. A interação entre o desenho do trabalho e as preferências do indivíduo acrescentou explicação ao modelo, com exceção do suporte social. Conclui-se que, a depender do tipo de característica, é preciso analisar a preferência do trabalhador ao se promover redesenho do trabalho ou para fazer a alocação de pessoal.
\end{abstract}

Keywords: Person-job fit, work engagement, polynomial regression

\begin{abstract}
The individual's needs play a relevant role in the relationship between work characteristics and engagement, but they are little explored. This study aims to analyze the interaction between individual and context to predict engagement, testing hypotheses derived from Self-Determination Theory and fit studies. A sample of 341 workers responded to the Engagement at Work and Work Design scales. Confirmatory factor analysis, polynomial regression, and response surface analysis were used to test the hypotheses. Knowledge characteristics, autonomy, and social support significantly explained the level of engagement. The interaction between work characteristics and the worker's desire added explanation to the model, except for social support. In conclusion it is necessary to analyze the workers' desire for a given characteristic to develop work designs that promote higher engagement.
\end{abstract}

¿Cuánto más, mejor? El efecto de la compatibilidad persona-trabajo en el engagement.

\section{Resumen}

Las necesidades del individuo tienen importante papel en la relación entre características del trabajo y engagement, pero son poco exploradas. Este estudio analizó la interacción entre el individuo y el contexto para predecir el engagement, probando hipótesis derivadas de la teoría de la autodeterminación y de los estudios de fit. La muestra de 341 trabajadores respondió las escalas de Engagement y Diseño del Trabajo. Se realizaron análisis factoriales confirmatorios, regresiones polinomiales y análisis de superficie de las respuestas para comprobar las hipótesis. Las características del conocimiento, autonomía y soporte social en el trabajo explicaron significativamente el nivel de engagement. La interacción entre las características del trabajo y el deseo del trabajador añadió explicación al modelo, con excepción del soporte social. Se concluye que, a depender del tipo de característica, es necesario examinar el grado de deseo del trabajador por aquella característica para desarrollar dibujos del trabajo que promuevan más engagement.

\footnotetext{
Endereço para correspondência:

ICC Sul. Instituto de Psicologia, Sala AT-013, Brasília (DF), CEP 70910-900. E-mail: porto.juliana@gmail.com

Como citar este artigo:

Porto, J. B., Oliveira-Silva, L. C., \& Martins, E. C. (2019). Quanto mais melhor? O efeito da compatibilidade pessoa-trabalho no engajamento. Revista Psicologia: Organizações e Trabalho, 19(4), 827-835. doi: 10.17652/rpot/2019.4.17418
} 
A busca pelos fatores que ajudam a fomentar estados psicológicos positivos nos indivíduos já é considerada tradicional no âmbito das humanidades. Uma vez que os estudos do campo da Psicologia Positiva têm, ao longo do tempo, ganhado cada vez mais adeptos e encontrado evidências continuamente consistentes, considera-se ponto pacífico a atenção a certas variáveis, seja no âmbito da vida em geral ou no contexto de trabalho. Neste sentido, o engajamento no trabalho representa uma das variáveis deste campo de estudo, cujo interesse, há alguns anos, extrapolou o ambiente acadêmico e tomou conta do mundo corporativo, chegando a ser considerado quase como uma "obsessão" dos departamentos de recursos humanos/gestão de pessoas das grandes organizações (Stairs \& Galpin, 2013).

O engajamento tem adquirido relevância no cenário organizacional ao ser associado às métricas financeiras (rentabilidade da empresa) e de clientes (satisfação e reputação da empresa) (Schneider, Hornung, Weigl, Glaser, \& Angerer, 2017), à maiores índices de produtividade e menores de rotatividade (Harter \& Blacksmith, 2013), assim como à maior satisfação, comprometimento organizacional, saúde mental, e aumento dos comportamentos extra papel e desempenho (Schaufeli, 2013). Devido a tal relevância, as pesquisas têm buscado identificar fatores influenciadores do engajamento, indo desde questões internas ao indivíduo até os aspectos externos, relativos aos contextos organizacionais e de trabalho.

Desde seus primórdios, os estudos sobre engajamento conduzidos por Kahn (1990) apontaram que as pessoas tendem a se engajar mais no trabalho quando encontram sentido nas atividades, se sentem seguros com o que fazem e conseguem transformar sua energia em bom desempenho. De especial interesse no presente estudo são as relações entre engajamento e desenho do trabalho (Work Design - WD), sendo este último indicado como um dos fatores que consistentemente influencia uma gama de comportamentos, atitudes e cognições dos indivíduos nos contextos laborais (Cordery \& Parker, 2012). No tocante às atitudes, especificamente, foram encontradas relações entre o WD e satisfação, envolvimento e comprometimento, construtos análogos ao engajamento (Brown, 1996; Dormann \& Zapf, 2001; Fedor, Cladwell, \& Herold, 2006).

O clássico estudo de Hackman e Oldham (1980) sobre as cinco principais características do trabalho - variedade, identidade e significado das tarefas, autonomia e feedback - já previa a influência destas na motivação e satisfação no trabalho. Para Stairs e Galpin (2013), apesar da antiguidade dos achados de Hackman \& Oldham (1980), as características apontadas não devem ser ignoradas no tocante à sua influência em estados psicológicos, principalmente ao se considerar o engajamento em seu sentido mais amplo.

As pesquisas apontam para a influência das características do trabalho na promoção do engajamento (Brown, 1996; Dormann \& Zapf, 2001; Fedor et al., 2006). Entretanto, ainda é necessário analisar o papel do indivíduo nesta relação e explorar o efeito da satisfação das preferências individuais em relação ao trabalho (Harter \& Blacksmith, 2013). Neste sentido, destaca-se a importância de analisar o quanto a compatibilidade, ou fit, entre as preferências do indivíduo e as características do WD contribuem para o aumento do engajamento. Logo, este estudo tem como objetivo analisar a predição da interação entre a característica do trabalho e as preferências do indivíduo no engajamento, testando hipóteses derivadas da teoria da autodeterminação (Self-Determination Theory - SDT) e dos estudos de fit.

\section{Engajamento e WD: O Papel da SDT e do Fit}

A compreensão de quais características do trabalho promovem atitudes positivas em quem trabalha tem ganhado cada vez mais destaque tanto na literatura nacional quanto internacional (e.g. Ferreira \& Mendonça, 2012; Linley, Harrington, \& Garcea, 2013;). Entretanto, no que diz respeito aos modelos de WD, o alto número de variáveis, sua diversidade e as interações entre as mesmas têm dificultado as pesquisas, que se esforçam para isolar os fatores e compreender quais deles exercem maior impacto em resultados positivos no trabalho. Dentre estes, o engajamento tem recebido destaque por enfatizar que os indivíduos podem extrair o melhor do trabalho, ao mesmo tempo que as organizações obtêm os meIhores resultados possíveis (Stairs \& Galpin, 2013).

De forma geral, o conceito de engajamento tem sido associado a felicidade no trabalho (Sender \& Fleck, 2017), sendo também compreendido como "paixão pelo trabalho" (Truss, Alfes, Delbridge, Shantz, \& Soane, 2013) ou quando alguém é completamente "envolvido e entusiasmado" com o próprio trabalho (Harter \& Blacksmith, 2013). Em termos operacionais, Schaufeli, Salanova, González-Romá e Bakker (2002) definem o engajamento como um estado psicológico positivo relacionado ao trabalho, e não à organização, de natureza afetivo-cognitiva, que se manifesta por meio da dedicação, do vigor e da absorção ao trabalho. A análise do engajamento pode ser realizada em duas dimensões: uma que indica o nível de prazer e outra que indica o nível de ativação (Bakker, Demerouti, \& Xanthopoulou, 2012; Schaufeli, 2013). O engajamento, no caso, é considerado um estado no qual o indivíduo vivencia altos níveis de prazer relacionado ao trabalho e, ao mesmo tempo, apresenta altos níveis de ativação diante do trabalho.

O WD se apresenta como um potencial antecedente de engajamento, uma vez que sua abrangência conceitual implica no estudo, criação e modificação da composição, conteúdo, estrutura e ambiente dentro do qual empregos e papéis de trabalho são definidos (Morgeson \& Humphrey, 2006). Logo, o WD é compreendido como uma variável dinâmica que pode ser impactada por mudanças contextuais, sejam elas sociais ou organizacionais (Cordery \& Parker, 2012). Um dos objetivos das proposições acerca do WD consiste em relacionar o trabalho com o ambiente que o cerca. Conceptualizações sobre o tema envolvem majoritariamente as características do trabalho, que têm sido definidas como atributos da tarefa, do trabalho e do ambiente social e organizacional (Morgeson \& Humphrey, 2006).

Embora atualmente a perspectiva adotada para a compreensão do WD apresente um enfoque integrado e interdisciplinar, abordando aspectos mecanicistas, biológicos, percepto-motores e relacionais (Parker, Morgeson, \& Johns, 2017), observa-se que a ênfase no fator motivacional não foi superada. Para Cordery e Parker (2012), a abordagem interdisciplinar deveria, a princípio, encorajar pesquisadores e profissionais a considerar que o WD deveria ir além de questões motivacionais e de desempenho. Porém, a realidade é que a as características motivacionais representam a faceta mais investigada da teoria, sendo apontada como aquela que mais reflete a complexidade do trabalho (Morgeson \& Humphrey, 2006). Isto ocorre, principalmente no âmbito da Psicologia Organizacional e do Trabalho, porque um dos princípios básicos da área é a possibilidade de que trabalho pode ser enriquecido de maneira a se tornar mais satisfatório e motivador, sendo as origens dos estudos do WD na psicologia baseados no fator motivacional (Cordery \& Parker, 2012).

A importância das características sociais e contextuais não deve ser deixada de lado. Humphrey, Nahrgang e Morgeson (2007), por exemplo, encontraram evidências de que as características 
motivacionais representam fortes preditores de desempenho, satisfação e comprometimento, porém o acréscimo de características sociais e do contexto de trabalho aumentaram o grau e a amplitude de predição. Tais evidências contribuíram para a expansão das características do trabalho, que passam a incluir três categorias de fatores associados ao WD: motivacional, social e contextual (Humphrey et al., 2007).

Uma das teorias que contribui para a compreensão das características do trabalho que mais afetam as atitudes positivas dos trabalhadores é a da SDT (Deci \& Ryan, 2000). Conhecida como umas das mais respaldadas teorias de motivação da contemporaneidade, tem sido associada por Gagné e Deci (2005) tanto à teoria das características do trabalho de Hackman e Oldham (1980), considerada o embrião dos modelos de WD (Cordery \& Parker, 2012).

A premissa básica da SDT reside na questão da motivação intrínseca versus extrínseca, sustentando que a compreensão da motivação humana se baseia nas necessidades universais de competência, autonomia e relacionamento (Deci \& Ryan, 2000). No tocante às aplicações da teoria ao contexto laboral, estudos como o de Gagné, Senécal e Koestner (1997) admitem que as características do trabalho se relacionam com motivação; entretanto, enfatiza-se a importância de aspectos relacionais, como o estilo interpessoal de supervisores e gerentes (Gagné \& Deci, 2005). A partir das evidências que associam a SDT ao engajamento no trabalho (e.g. Meyer \& Gagné, 2008) e em estudantes (e.g.; Leal, Miranda, \& Carmo, 2013), compreende-se ser plausível utilizar tal teoria como base para selecionar e agrupar as dimensões do WD que podem gerar maior engajamento. Considerando o modelo proposto por Morgeson e Humphrey (2006), a dimensão de características sociais poderia ser associada à necessidade de relacionamento da SDT; a autonomia, oriunda da dimensão de características da tarefa, se relacionaria com a necessidade de autonomia da SDT; por fim, a dimensão do conhecimento poderia ser associada à necessidade de competência proposta pela SDT. A definição de cada dimensão e seu possível equivalente na SDT estão dispostos na Figura 1.

\section{Dimensão WD \\ (Morgeson \& Humphrey, 2006)}

Características sociais: Refletem o quanto Necessidade de relacionaum trabalho é realizado em um ambiente mento: Busca por pertencer social. Compreende os aspectos sociais e a um grupo ou uma organiinterpessoais do trabalho. Subdivide-se em zação, visando a socialização suporte social, interdependência, interação e a interação social na aprenfora da organização e feedback dos outros. dizagem e realização de atividades.

Autonomia (características da tarefa): Necessidade de autonomia: Compreende o quanto é cedido ao indiví- Busca pelo controle das próduo liberdade, independência e critérios prias ações e da situação, para planejamento do trabalho, tomada de independentemente dos redecisões e escolha de métodos utilizados sultados a serem alcançados, para executar as tarefas.

Conhecimento: Tipos de conhecimentos, Necessidade de competênhabilidades e competências que o trabalho cia: Busca pelo domínio de exige que o indivíduo em sua função exer- tarefas e desenvolvimento de ça. Representa, junto com as tarefas, o blo- maestria, através do contato co das características motivacionais, que com novos desafios e oportutem como princípio básico o enriquecimen- nidades para demonstrar coto do trabalho. Subdivide-se em: complexi- nhecimento e adquirir novas dade da tarefa, processamento de informa- habilidades.

ção, resolução de problemas, variedade de competências e especialização.

Figura 1. Quadro de Dimensão do WD e seus equivalentes na teoria da SDT

Ademais, a literatura indica que, de fato, tais dimensões do WD podem gerar mudanças atitudinais nos trabalhadores de forma a afetar fatores como o engajamento (Petrou, Demerouti, Peeters, Schaufeli, \& Hetland, 2012). Revisão da literatura indica que fatores como oportunidades de desenvolvimento, feedback, autonomia, variedade de competências, liderança transformacional, justiça e suporte social de colegas e supervisores contribuem para aumentar o engajamento dos trabalhadores (Schaufeli, 2012); bem como empregados demonstram-se mais motivados e satisfeitos em empregos com altos requisitos de solução de problemas que oferecem uma oportunidade para os funcionários se apresentarem em situações novas e desafiadoras nas quais podem exercer e reforçar seu senso de competência no trabalho (Deci \& Ryan, 2000). Aparentemente, o aumento das exigências de conhecimento também torna o trabalho mais intrinsecamente motivador e promove atitudes positivas. Tal fenômeno pode ser explicado pelo processo motivacional proposto pelo Modelo de Recursos e Demandas (JDR) descrito por Schaufeli (2017), que prevê que, em contextos nos quais os recursos são abundantes e as exigências (demandas) são reduzidas, o processo psicológico desencadeado é o engajamento. Por outro lado, é fundamental ressaltar que o modelo JD-R propõe também um processo via burnout, quando se aumentam as exigências (demandas) e se limitam os recursos (Bakker, Demerouti, \& Sanz-Vergel, 2014). Sendo assim, ressalta-se a importância da consideração das necessidades do indivíduo e da compatibilidade entre o que o indivíduo prefere e o que o trabalho fornece.

Os comportamentos de redesenho e as possibilidades de adequação entre o trabalho e o trabalhador têm como foco o aumento da compatibilidade entre o indivíduo e o seu trabalho (Tims, Bakker, \& Derks, 2012). A busca por tal adequação seria capaz de modificar a identidade do trabalho e revisar o significado deste para o trabalhador (Wrzesniewski \& Dutton, 2001). Em linha com esse pensamento, Chinelato, Ferreira e Valentini (2015) encontraram correlações positivas entre os comportamentos de redesenho e o engajamento no trabalho, assim como Petrou et al. (2012), que perceberam uma associação entre a flutuação dos comportamentos de redesenho e a flutuação do engajamento no trabalho com o passar dos dias. Por fim, Schaufeli, Bakker e Van Rhenen (2009) identificaram que mudanças em recursos de trabalho predizem engajamento após um ano. Portanto, nota-se que além de haver indícios da relação entre WD e engajamento, a capacidade do indivíduo de alterar essas características influencia os níveis de engajamento de maneira positiva e duradoura.

As teorias de compatibilidade pessoa e ambiente ressaltam a relevância da interação da pessoa com o contexto para compreender os fenômenos psicológicos. As pesquisas sobre o tema fazem uma combinação entre os indivíduos e os vários níveis da atividade laboral, envolvendo não só o trabalho, como também a organização, os grupos de trabalho, os supervisores e até mesmo questões vocacionais (Kristof-Brown, Zimmerman, \& Johnson, 2005). Entretanto, a compatibilidade pessoa-trabalho representa um dos tipos de fit mais investigados, sendo definida como a relação entre as características de uma pessoa e as do trabalho ou tarefas que são realizadas no local de trabalho (Sekiguchi \& Huber, 2011).

Duas conceptualizações básicas descrevem o fit entre pessoa e trabalho (Edwards, 1991). A primeira é relativa à interação entre as exigências e as capacidades do indivíduo, de forma que o conhecimento, as habilidades e as capacidades são avaliadas de acordo com aquilo que o trabalho exige, sendo conhecida como demanda-habilidade (D-H). A segunda, por sua vez, ocorre quando as necessidades, desejos ou preferências das pessoas são atendidas pelo trabalho que realizam, perspectiva conhecida como necessidade-suporte ou N-S (Chen, Yen, \& Tsai, 2014; Lin, Yu, \& Yi, 2014). Esta última abordagem foi utilizada no presente estudo para a compreensão do fit, pois o redesenho visa alterar o nível de demandas e recursos de forma a adequar o trabalho às necessidades, 
preferências, motivações, paixões, valores e competências do indivíduo (Tims et al., 2012).

A teoria de compatibilidade no trabalho contribui para o consenso de que o conhecimento acerca dos desejos, necessidades e preferências do trabalhador é fundamental para intervir nos contextos laborais de maneira a aumentar os comportamentos e as atitudes positivas no trabalho. Pesquisas na área têm reforçado tal premissa, evidenciando que o fit pessoa-trabalho se correlaciona positivamente com certas atitudes e comportamentos por parte dos trabalhadores, como é o caso da satisfação, do comprometimento, da retenção, comportamentos de cidadania e do desempenho (Sekiguchi \& Huber, 2011), assim como do engajamento (Lauver \& Kristof-Brown, 2001). Adicionalmente, há indícios de que quanto maior a compatibilidade entre o indivíduo e o seu ambiente de trabalho, mais positivos serão os resultados no trabalho (KristofBrown et al., 2005; Sulistiowati, Komari, \& Dhamayanti, 2018).

Apesar de serem vários os estudos que apontam para as relações entre engajamento e o WD, tais como suporte social, feedbacks, variedade de tarefas, autonomia e oportunidades de aprendizagem (Bakker \& Demerouti, 2008), ainda são escassos aqueles que analisam o papel do fit entre as preferências do indivíduo e tais características. De forma geral, as pesquisas apontam que funcionários com melhor ajuste em relação ao seu trabalho apresentam maior engajamento no trabalho (ver Chen et al., 2014; Lu, Wang, Lu, Du, \& Bakker, 2014; Shuck, Reio, \& Rocco, 2011), porém não há consenso em relação às condições nas quais tais fatores afetam o engajamento.

As teorias de fit e a SDT conversam entre si na medida em que a presença do fit se relacionaria com indivíduos que são capazes de satisfazer suas necessidades psicológicas, que segundo a SDT consistem em autonomia, relacionamento e competência (Greguras \& Diefendorff, 2009). Entretanto, ao se isolar as premissas da SDT, compreende-se que não seria o grau de preferência ou necessidade o fator mais importante na previsão de resultados positivos, e sim a satisfação destas três necessidades (Deci \& Ryan, 2000). Tal satisfação poderia ser alcançada, por sua vez, através da presença ou não de certas características no trabalho. A preferência por um alto salário, por exemplo, não representaria uma necessidade porque é um motivo aprendido, não essencial nem universal, além de não necessariamente gerar resultados favoráveis (Greguras \& Diefendorff, 2009). Em outras palavras, a mera satisfação dessa vontade do indivíduo no trabalho poderia levar a resultados tanto positivos quanto negativos. O princípio central da SDT é que a satisfação de uma necessidade psicológica sempre leva a resultados favoráveis, porque é ilógico para a satisfação de uma necessidade - algo que é essencial - gerar resultados desfavoráveis (Deci \& Ryan, 2000).

Nesta concepção, uma vez que características do trabalho associadas a autonomia, relacionamento e competência estejam presentes, as necessidades psicológicas do indivíduo estariam satisfeitas, o que levaria a resultados favoráveis como o engajamento no trabalho. Apesar de considerar o indivíduo nessa relação, a teoria pressupõe que todos possuem essas necessidades e, portanto, quanto mais o contexto provê a satisfação dessas necessidades, mais o indivíduo apresenta resultados positivos. Sendo assim, o valor do fit entre o trabalho e as preferências do indivíduo seria irrelevante, pois não haveria variação no grau de necessidade do indivíduo, o que conduz à primeira hipótese a ser testada neste estudo:

H1: Em concordância com as premissas da SDT, as características do WD (autonomia, conhecimento e suporte social) predizem o engajamento no trabalho de forma linear e positiva, independentemente do grau de preferência do indivíduo. Cabe lembrar, ainda, que tanto a SDT quanto as teorias de fit enfatizam a relação entre indivíduos e contexto e assumem que atitudes positivas dependem do grau de correspondência entre fatores contextuais e atributos pessoais (Greguras, Diefendorff, Carpenter, \& Tröster, 2014). Contudo, proposições fundamentais da SDT têm sido colocadas em questão. Andersen, Chen e Carter (2000), por exemplo, sugeriram que indivíduos têm mais de três necessidades psicológicas fundamentais; Carver e Scheier (1999) questionaram a importância da necessidade de autonomia para todas as pessoas; Buunk e Nauta (2000) afirmam que não satisfazer algumas necessidades psicológicas pode na verdade aumentar o bem-estar (alguns funcionários podem preferir supervisão à autonomia); por fim, Pyszczynski, Greenberg e Solomon (2000) argumentam que a satisfação de necessidades particulares pode gerar conflitos entre si. Adicionalmente, as pesquisas de fit apontam que a compatibilidade tem valor incremental na explicação dos resultados individuais, apesar de haver evidências para o efeito independente de características contextuais; níveis ótimos de impacto nos resultados individuais são obtidos quando os indivíduos experienciam fit em atributos individualmente mais relevantes; e tanto a deficiência quanto o excesso de falta de fit são prejudiciais, mas a deficiência tende a ser mais prejudicial (van Vianen, 2018).

Estudos sobre fit pessoa-trabalho tem investigado mais frequentemente demandas, sobrecarga, insegurança no trabalho, promoção de oportunidades, ambiguidade de papéis, suporte do supervisor e autonomia (van Vianen, 2018). As pesquisas, em geral, apontam que os atributos do trabalho tendem a impactar nos resultados, mas que a combinação entre características do trabalho e das pessoas acrescentam explicação (van Vianen, 2018). A partir de tais questões, delineia-se a segunda hipótese deste estudo:

H2: Em concordância com as premissas das teorias de compatibilidade, o fit entre características do WD (autonomia, conhecimento e suporte social) e as preferências do indivíduo por essas características prediz significativamente o engajamento no trabalho. Para testar essas hipóteses, foi desenvolvido um estudo de corte transversal que avaliou o efeito do fit entre caraterísticas do trabalho e as preferência do indivíduo sobre o nível de engajamento, utilizando as técnicas mais adequadas para teste do fenômeno, a regressão polinomial e análises de gráficos de superfície (van Vianen, 2018). As características do trabalho testadas no modelo foram aquelas relacionadas à SDT, a saber: conhecimento, autonomia na organização do trabalho, autonomia no estabelecimento do método de trabalho e suporte social.

\section{Método}

\section{Participantes}

A amostra foi composta de 341 respondentes válidos que concordaram com o Termo de Consentimento Livre e Esclarecido e se declararam como atualmente empregados há mais de seis meses. Mais da metade dos respondentes se identificaram como do sexo feminino $(64,2 \%)$ e como solteiros $(60,1 \%)$, enquanto metade dos respondentes tinha menos de 30 anos de idade $(51,9 \%)$ e trabalhavam há, pelo menos, três anos e seis meses na empresa atual (49,9\%). Acerca da localização geográfica da amostra, a maior representatividade foi da região sudeste (54\%) e da região centro-oeste $(36,4 \%)$. Considerando as relações trabalhistas, maior parte da amostra era composta de pessoas com contratos individuais regulados pela CLT $(55,1 \%)$ e de estatutários/concursados $(17,9 \%)$, mas também participaram autônomos $(9,1 \%)$, estagiários $(7,3 \%)$, profissionais liberais $(4,7 \%)$ e outros $(5,9 \%)$. A área das empresas 
foi principalmente serviços $(49,9 \%)$ e o tipo de empresa predominante foram as empresas privadas $(63,6 \%)$, seguido pelas empresas públicas $(26,1 \%)$, ONG $(3,2 \%)$, Cooperativa $(1,5 \%)$ e não informaram $(5,6 \%)$. As empresas tinham, em sua maioria, mais de 500 empregados $(44,6 \%)$.

\section{Instrumentos}

Engajamento no trabalho. Versão reduzida e traduzida por Vazquez, Magnan, Pacico, Hutz e Schaufeli (2015) da Escala Utrecht de Engajamento no Trabalho (UWES-9). O instrumento é composto por nove itens, que se dividem teoricamente em três dimensões: vigor, dedicação e absorção ao trabalho. A escala do instrumento vai de 1 (nunca) a 5 (diariamente).

Exemplos: "Sou entusiasmado com meu trabalho", "Sinto orguIho do trabalho que faço", "Deixo-me levar pelo trabalho quando estou trabalhando".

WD. Versão reduzida e traduzida do Questionário de WDQ (Borges-Andrade, Peixoto, Queiroga, \& Pérez-Nebra, 2019). O instrumento original (Morgeson \& Humphrey, 2006) é composto por 77 itens, que se dividem teoricamente em quatro dimensões com 21 subdimensões, mas para este estudo foram utilizados apenas 28 itens, englobando três dimensões (características da tarefa, características sociais e características do conhecimento) com 9 subdimensões. Das características da tarefa, foram utilizadas integralmente três das sete subdimensões, foram elas: autonomia na organização do trabalho ( 3 itens), autonomia nos métodos de trabalho (3 itens) e variedade de tarefas (4 itens). Das características sociais, foram utilizadas integralmente três das cinco subdimensões, foram elas: suporte social (6 itens), interação fora da organização (4 itens) e feedback dos outros (3 itens). Das características do conhecimento, foi testada uma versão reduzida com itens que representavam três das cinco dimensões: processamento de informação ( 2 itens), solução de problemas ( 2 itens) e especialização ( 1 item).

Este instrumento possuía duas escalas de resposta, que iam de 1 (Discordo totalmente) a 5 (Concordo totalmente), uma para o WD atual e uma para o WD desejado. $O$ enunciado do instrumento orientava: "Para a característica ATUAL, pense em seu trabalho e em seu papel atual e assinale a opção correspondente à sua resposta. Para a característica DESEJÁVEL, pense o quanto você GOSTARIA que seu trabalho tivesse as características descritas (independentemente de o seu trabalho atual possuir ou não essa característica) e assinale a opção correspondente à sua resposta". Exemplos: "Os meus colegas de trabalho são amigáveis", "O meu trabalho exige a realização de um amplo conjunto de tarefas" e "O meu trabalho faz com que eu tenha que lidar, muitas vezes, com problemas que eu nunca tinha visto antes".

\section{Procedimentos de Coleta de Dados e Cuidados Éticos}

Os dados foram coletados de forma online na plataforma de formulários do Google por meio da divulgação em redes sociais e em disciplinas de nível superior por um grupo de pesquisadoras de Brasília, São Paulo e Minas Gerais. O projeto de pesquisa foi aprovado pelo Comitê de Ética (CAAE: 79450117.9.0000.5508 em 12/12/2017).). Houve apresentação do Termo de Consentimento Livre e Esclarecido (TCLE) a todos os respondentes e o prosseguimento ao preenchimento dos instrumentos só era possível após a concordância com o descrito pelo TCLE. A amostra obtida foi não probabilística e o estudo fez um levantamento de dados transversal. Não houve aleatorização dos itens e dos formulários e nem feedback aos respondentes após o preenchimento de todos os instrumentos.

\section{Procedimentos de Análise de Dados}

Para análise de dados, inicialmente foram realizadas análises fatoriais confirmatórias dos instrumentos, utilizando o MPlus. Para essas análises optou-se pelo método de estimação MLR, que é robusto à violação do pressuposto da distribuição normal (Satorra \& Bentler, 2001). Foram consideradas satisfatórias as escalas que atenderam a pelo menos dois dos seguintes critérios para avaliação dos modelos: CFI > 0,95 e SRMR < 0,08 ou RMSEA < 0,06 (Hu \& Bentler, 1999).

Em seguida foram realizadas regressões polinomiais e análises de superfície do padrão de respostas, uma vez que possibilitam o teste das proposições teóricas de fit de forma mais adequada por considerar os níveis de todas as variáveis e o grau de compatibilidade entre elas (van Vianen, 2018). A análise de superfície do padrão de respostas nos permite identificar o efeito tanto da compatibilidade quanto da incompatibilidade entre as percepções do WD atuais e desejados sobre o nível do engajamento de trabalhadores. Para isso, garantimos que as variáveis foram mensuradas em uma mesma escala numérica e compartilharam um mesmo domínio conceitual. Para essas análises foram seguidas as orientações de Humberg, Nestler e Back (2018), conforme descrito a seguir.

Antes de realizar as análises, foi inspecionada a distribuição da discrepância entre as duas variáveis antecedentes (WD atual $x$ desejado) para verificar se havia variabilidade suficiente na amostra para conduzir as análises seguintes. Nesta análise os escores foram padronizados e foi calculada a distribuição entre três grupos: 1) WD Atual maior que desejado; 2 ) indicadores de WD compatíveis; e, 3) WD desejado maior que atual. Para a análise dos pressupostos da regressão polinomial foram examinados os gráficos de resíduos e utilizado o indicador Durbin-Watson para avaliar o desvio de normalidade e o VIF (Fatores de inflação de variância) para avaliar a multicolinearidade. Os casos extremos multivariados identificados na regressão foram eliminados. Antes da regressão polinomial, as variáveis antecedentes foram centralizadas pela média do grupo. Para cada tipo de fit foi realizada uma análise de regressão no $\mathrm{R}$ utilizando o pacote RSA versão 0.9.12 (Schönbrodt \& Humberg, 2018). Após as análises de regressão, foram realizados gráficos tridimensionais para auxiliar na interpretação.

\section{Resultados}

\section{Análise das Propriedades Psicométricas}

As análises fatoriais confirmatórias foram realizadas para as 5 escalas: Engajamento no trabalho, WD conhecimento atual, WD conhecimento desejado, WDQ Atual e WDQ Desejado. Como os fatores de características do conhecimento foram alterados, foi realizada a análise fatorial destes itens em separado. Os índices de ajuste dessas análises podem ser observados na Tabela 1 . Todas as escalas apresentaram bons índices de ajuste e atenderam ao critério de corte, exceto a Escala de WD que atingiu o critério para um indicador e os demais ficaram próximos. A análise dos parâmetros e resíduos indicou que um item de suporte social apresentava baixa carga fatorial, assim optou-se por retirá-lo e o novo modelo apresentou melhores índices de ajuste. A confiabilidade composta para os fatores foram: Engajamento no trabalho $=0,99 ; \mathrm{WD}$ Conhecimento Atual =0,99; WD Conhecimento Desejado $=0,98$; WD Organização do trabalho Atual $=0,98$; WD Organização dos métodos de trabalho Atual $=0,99$; WD Suporte Social Atual $=0,98$; WD Organização do trabalho Desejado $=0,98$; WD Organização dos métodos de trabalho Desejado =0,98; WD Suporte Social Desejado $=0,99$. 
Tabela 1

Indicadores de ajuste da modelagem por equações estruturais das escalas utilizadas

\begin{tabular}{lcccc}
\hline Escala & $c^{2}(g l)$ & CFI & RMSEA (IC 90\%) & SRMR \\
\hline $\begin{array}{l}\text { Engajamento no trabalho } \\
\text { (Ferreira et al., 2016) }\end{array}$ & $55,64(23)$ & 0,98 & $0,07(0,04-0,09)$ & 0,03 \\
$\begin{array}{l}\text { WD Conhecimento } \\
\text { Desejado }\end{array}$ & $11,21(5)$ & 0,98 & $0,06(0,01-0,11)$ & 0,02 \\
WD Conhecimento Atual & $32,61(5)$ & 0,95 & $0,12(0,09-0,17)$ & 0,03 \\
WDQ Atual -6 fatores & $569,34(215)$ & 0,91 & $0,07(0,06-0,08)$ & 0,06 \\
$\begin{array}{l}\text { WDQ Atual - 6 fatores sem } \\
\text { item 19 }\end{array}$ & $414,76(194)$ & 0,94 & $0,06(0,05-0,07)$ & 0,06 \\
$\begin{array}{l}\text { WDQ Desejado-6 fatores } \\
\text { WDQ Desejado -6 fatores }\end{array}$ & $551,07(215)$ & 0,91 & $0,07(0,06-0,08)$ & 0,06 \\
sem item 19 & $415,90(194)$ & 0,94 & $0,06(0,05-0,07)$ & 0,05 \\
\hline
\end{tabular}

Nota. Х2: qui-quadrado; gl: graus de liberdade; CFI: Comparative Fix Index RMSEA: Root Mean Square Error of Approximation; SRMR: Standardized Root Mean Square Residual.

\section{Análise do Efeito da (In)compatibilidade do WD sobre o Engajamento}

Após as análises das propriedades psicométricas das escalas, foram calculadas a distribuição das discrepâncias para avaliar se havia variabilidade de respostas. Os resultados indicaram que há variação de respostas suficiente, mais de $10 \%$ de indivíduos em cada grupo. A Tabela 2 apresenta esses resultados.

Tabela 2

Frequência dos escores de WD Atual que são maiores, compativeis ou menos do que o WD Desejado

\begin{tabular}{|c|c|c|c|c|}
\hline \multirow[b]{2}{*}{$\begin{array}{l}\text { Caracte- } \\
\text { rísticas do } \\
\text { WD }\end{array}$} & \multirow[b]{2}{*}{$\begin{array}{l}\text { Dados descri- } \\
\text { tivos }\end{array}$} & \multicolumn{3}{|c|}{ Grupo conforme grau de compatibilidade } \\
\hline & & $\begin{array}{l}\text { WD Atual > } \\
\text { WD Desejado }\end{array}$ & $\begin{array}{l}\text { WD Atual = } \\
\text { WD Desejado }\end{array}$ & $\begin{array}{l}\text { WD Desejado } \\
>\text { WD Atual }\end{array}$ \\
\hline \multirow{3}{*}{$\begin{array}{l}\text { Conheci- } \\
\text { mento }\end{array}$} & Porcentagem & $24 \%$ & $55 \%$ & $21 \%$ \\
\hline & $\begin{array}{l}\text { Média não- } \\
\text {-padronizada } \\
\text { WD Atual }\end{array}$ & 3,85 & 4,11 & 2,99 \\
\hline & $\begin{array}{c}\text { Média não-pa- } \\
\text { dronizada WD } \\
\text { Desejado }\end{array}$ & 3,39 & 4,20 & 4,30 \\
\hline \multirow{3}{*}{$\begin{array}{l}\text { Autonomia } \\
\text { na organi- } \\
\text { zação do } \\
\text { trabalho }\end{array}$} & Porcentagem & $39 \%$ & $34 \%$ & $27 \%$ \\
\hline & $\begin{array}{l}\text { Média não- } \\
\text {-padronizada } \\
\text { WD Atual }\end{array}$ & 3,50 & 3,76 & 2,13 \\
\hline & $\begin{array}{l}\text { Média não-pa- } \\
\text { dronizada WD } \\
\text { Desejado }\end{array}$ & 3,42 & 4,35 & 4,37 \\
\hline \multirow{3}{*}{$\begin{array}{l}\text { Autono- } \\
\text { mia de } \\
\text { método de } \\
\text { trabalho }\end{array}$} & Porcentagem & $39 \%$ & $34 \%$ & $27 \%$ \\
\hline & $\begin{array}{l}\text { Média não- } \\
\text {-padronizada } \\
\text { WD Atual }\end{array}$ & 3,45 & 3,66 & 2,12 \\
\hline & $\begin{array}{l}\text { Média não-pa- } \\
\text { dronizada WD } \\
\text { Desejado }\end{array}$ & 3,41 & 4,30 & 4,35 \\
\hline \multirow{3}{*}{$\begin{array}{l}\text { Suporte } \\
\text { social }\end{array}$} & Porcentagem & $36 \%$ & $40 \%$ & $25 \%$ \\
\hline & $\begin{array}{l}\text { Média não- } \\
\text {-padronizada } \\
\text { WD Atual }\end{array}$ & 3,79 & 4,13 & 3,11 \\
\hline & $\begin{array}{c}\text { Média não-pa- } \\
\text { dronizada WD } \\
\text { Desejado }\end{array}$ & 3,79 & 4,53 & 4,52 \\
\hline
\end{tabular}

Por fim, foram realizadas regressões polinomiais para análise dos efeitos da compatibilidade sobre o nível de engajamento no trabalho. Para cada par de compatibilidade (desejado e atual) foi realizada uma análise de regressão. Para todos os modelos, a característica do trabalho explicou significativamente o nível de engajamento. A interação entre característica e o desejo do trabalhador acrescentou explicação ao modelo para todos modelos, exceto para suporte social. A Tabela 3 apresenta os resultados das regressões e a Figura 2 apresenta os gráficos de superfície para todas as variáveis.

A regressão polinomial com análise de gráficos de superfície para a variável características do conhecimento encontrou efeito marginalmente significativo da congruência entre WD atual e desejado sobre o engajamento. Apesar da posição do eixo principal atender aos critérios da congruência $\left(p_{10}\right.$ é não significativo e o intervalo de confiança de $p_{11}$ não exclui o 1) e o efeito da superfície acima da linha de incongruência (LOIC) ter um formato de U invertido ( $a_{4}$ é negativo e significativo), o pressuposto de que este $U$ invertido é maximizado no ponto de congruência ( $a_{3}$ é não significativo) se considerado o critério de $p<0,01$, não foi obtido. Por outro lado, para $\mathrm{p}<0,05$ esse pressuposto é satisfeito. Adicionalmente, os resultados indicam uma combinação entre o efeito da congruência e o efeito principal curvilinear ( $a_{1}$ e $a_{2}$ são positivos e significativos). Isso quer dizer que tanto a congruência quanto as variáveis preditoras estão relacionadas com engajamento. $O \mathrm{R}^{2}$ do modelo foi 0,20 , $\mathrm{p}<0,001$. Assim, temos apoio para $\mathrm{H} 2$.
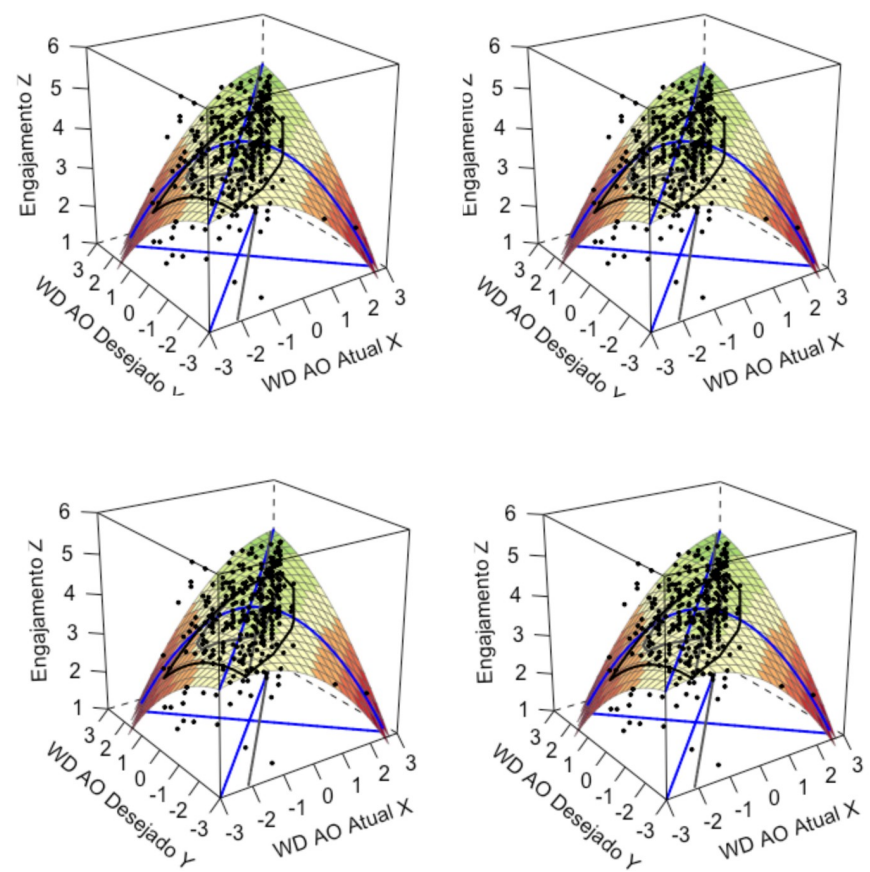

Figura 2. Gráficos de superfície para as regressões polinomiais de características de conhecimento do desenho do trabalho (WD CO), autonomia da organização do trabalho (WD AO), autonomia de método do trabalho (WD AM) e suporte social (WD SS) sobre o Engajamento no trabalho.

Para as variáveis WD autonomia na organização do trabalho e WD autonomia no método de trabalho, os resultados indicam que há um efeito da congruência ampla. A posição do eixo principal atende aos critérios da congruência ( $p_{10}$ é não significativo e o intervalo de confiança de $p_{11}$ não exclui o 1 ), o efeito da superfície acima da LOIC tem um formato de $U$ invertido e o pressuposto de que este $U$ invertido é maximizado no ponto de congruência foi obtido para $p<0,01$. Adicionalmente, os resultados indicam uma combinação entre o efeito da congruência e o efeito principal ( $a_{1}$ é positivo e significativo e $a_{2}$ é não significativo). Isso quer dizer que para pessoas com o mesmo grau de compatibilidade de autonomia, haverá mais engajamento para aquelas com níveis mais altos dos preditores (mais autonomia atual e desejada). Para WD autonomia na organização do trabalho, o $\mathrm{R}^{2}$ foi $0,157, \mathrm{p}<0,001$ e para autonomia 
Tabela 3

Resultados das análises de superfície para a predição de fit de características do trabalho sobre o engajamento

\begin{tabular}{|c|c|c|c|c|c|c|c|c|c|c|c|c|}
\hline \multirow{3}{*}{ Fit } & \multicolumn{6}{|c|}{ Parâmetros estimados do modelo de regressão } & \multirow{2}{*}{\multicolumn{2}{|c|}{$\begin{array}{l}\text { Posição do primeiro } \\
\text { eixo principal }\end{array}$}} & \multicolumn{4}{|c|}{ Formato da superfície } \\
\hline & \multicolumn{6}{|c|}{$\mathrm{Z}=b_{0}+b_{1} \mathrm{X}+b_{2} \mathrm{Y}+b_{3} \mathrm{X}^{2}+b_{4} \mathrm{XY}+b_{5} \mathrm{Y}^{2}$} & & & \multicolumn{2}{|c|}{ LOC } & \multicolumn{2}{|c|}{ LOIC } \\
\hline & $b_{0}$ & $b_{1}$ & $b_{2}$ & $b_{3}$ & $b_{4}$ & $b_{5}$ & $p_{10}$ & $p_{11}$ & $a 1$ & $a 2$ & $a 3$ & $a 4$ \\
\hline WD CO & $3,68 * *$ & $0,26 * *$ & $-0,20$ & $-0,16$ & $0,13 * *$ & $-0,30$ & $-0,66$ & $0,78^{\mathrm{a}}$ & $0,41 * *$ & $0,13^{*}$ & $0,40 *$ & $-0,47 * *$ \\
\hline WD AO & $3,94 * *$ & $0,18^{* *}$ & 0,01 & $-0,13^{* *}$ & $0,23 * *$ & $-0,09$ & $-0,45$ & $1,19^{a}$ & $0,19 * *$ & 0,02 & 0,17 & $-0,45 * *$ \\
\hline WD AM & $4,01 * *$ & $0,36^{* *}$ & 0,18 & $-0,04 * *$ & $0,34 * *$ & 0,12 & $-1,13$ & $1,86^{a}$ & $0,29 * *$ & 0,08 & 0,22 & $-0,35 * *$ \\
\hline WD SS & $3,83 * *$ & $0,89 * *$ & 0,04 & 0,20 & $-0,00 *$ & $0,52^{*}$ & $-41,01$ & $-2,22$ & $0,50 * *$ & 0,12 & $0,89 * *$ & $0,62 * *$ \\
\hline
\end{tabular}

Nota. $n=341$; WD CO = Características de conhecimento; WD AO = Características de autonomia de organização do trabalho; WD AM = Características de autonomia do método de trabalho; WD SS = Características de suporte social; $p_{10}=$ intercepto do eixo principal; $p_{11}=$ inclinação (slope) do eixo principal; LOC = Linha de congruência; LOIC = Linha de incongruência. ${ }^{a}$ O intervalo de $95 \%$ confiança não exclui o $1{ }^{*} p<0,05,{ }^{* *} p<0,01$

no método de trabalho, $R^{2}=0,196, p<0,001$. Novamente, os resultados apoiam $\mathrm{H} 2$.

Por último, para a variável suporte social, não foi verificado um efeito da congruência, uma vez que $p_{11}$ exclui o 1,0. Isso significa que a posição da linha que contém todas as combinações de congruência das variáveis preditoras observadas não corresponde à linha esperada. Entretanto, a variável suporte social atual, ou seja, aquele percebido pelos trabalhadores como presente no seu ambiente de trabalho, apresenta efeito direto e significativo. Esses resultados oferecem apoio para $\mathrm{H} 1$.

\section{Discussão}

Inicialmente, foram avaliadas as propriedades psicométricas das escalas utilizadas no estudo. Os resultados apresentados evidenciam bons indicadores e corroboram os estudos anteriores (Borges-Andrade et al., 2019; Ferreira et al., 2016). Assim, foi possível prosseguir para avaliar os efeitos do fit sobre o engajamento no trabalho.

Este estudo se propôs a investigar duas hipóteses contraditórias, uma advinda da SDT, que indica que a interação entre o WD e a preferência do indivíduo não são relevantes para explicar o grau de engajamento $(\mathrm{H} 1)$ e a segunda advinda das teorias de fit que afirma que essa interação acrescenta à explicação do fenômeno (H2) Embora os resultados apresentem apoio para as duas hipóteses, isto ocorre a depender da variável investigada. A importância de se considerar os efeitos diferenciados de cada tipo de característica do WD no fit está em sintonia com a revisão realizada por van Vianen (2018). Isso reforça a importância da teoria de WD (Morgeson \& Humphrey, 2006) que permite categorizar e compreender os seus efeitos de forma global.

A H1 recebeu apoio para a relação entre suporte social e engajamento. Os resultados demonstraram que a congruência não acrescentou explicação após o efeito direto do suporte. Esses resultados estão em sintonia com a revisão de Schaufeli (2012) e Bakker et al. (2014), que já apontava o efeito positivo do suporte social para o engajamento. $O$ suporte social, conforme o modelo de demandas e recursos do trabalho - JD-R, funciona como um recurso do trabalho de nível social que ajuda a alcançar as metas de trabalho ou a reduzir os efeitos nocivos das demandas de trabalho. Por outro lado, essa característica, diferentemente daquelas de conhecimento e autonomia, independe do desejo do trabalhador por ter suporte ou pode-se entender que, segundo a SDT, ele supre uma necessidade básica do indivíduo e quanto mais está presente no ambiente de trabalho, melhor é.

Em contrapartida, os resultados para autonomia e conhecimento indicam um efeito do fit, dando apoio à $\mathrm{H} 2$. Os resultados indicam que, no geral, os resultados positivos sobre o engajamento tendem a ser maiores quando há fit. No caso das características de conhecimento e de autonomia, há um efeito da congruência combinado com um efeito principal das variáveis preditoras, o que acarreta um formato curvilinear na linha acima do LOC. Isso indica que não apenas a congruência tem efeito, mas também o nível de cada variável preditora. Ao observar os gráficos é possível verificar que quando a pessoa valoriza em alto grau e o seu trabalho apresenta autonomia e conhecimento, o efeito é maior para o nível de engajamento. Esses achados podem explicar as relações em formato de $U$ para variáveis relativas ao WD encontradas anteriormente na literatura, especialmente para autonomia. Tangirala e Ramanujam (2008), por exemplo, encontraram uma relação em formato de $U$ entre as variáveis percepção de controle pessoal (que possui o componente de autonomia) e o comportamento de cidadania organizacional de expressão de ideias, opiniões e preocupações em relação ao trabalho. Considerando o resultado obtido de $\mathrm{U}$ invertido para as variáveis WD autonomia na organização do trabalho e WD autonomia no método de trabalho, De Jonge e Schaufeli (1998) evidenciaram a mesma relação entre presença de autonomia e exaustão emocional. Os estudos anteriores podem agora ser compreendidos por um efeito da interação entre a presença das características no WD e as preferências do indivíduo. Tal aspecto já havia sido sinalizado em estudo anterior (De Jonge \& Schaufeli, 1998), sendo agora apresentadas evidências.

\section{Considerações Finais}

O objetivo deste estudo foi analisar a predição da interação entre a característica do trabalho e as preferências do indivíduo no engajamento, testando hipóteses derivadas da SDT e dos estudos de fit. Os resultados permitem concluir que a depender do tipo de característica do trabalho que é investigada, o fit pode ou não ser relevantes. As características de conhecimento e de autonomia da tarefa apontam para a necessidade de compreender a congruência entre a preferência do indivíduo e a sua presença no trabalho para influenciar o seu engajamento. Por outro lado, suporte social parece ser um recurso do trabalho que quanto mais se tem, melhor é o impacto sobre o engajamento. Assim, este estudo contribui tanto teoricamente quanto para a prática do profissional.

Teoricamente, o estudo aponta para limitações da SDT (Deci \& Ryan, 2000), ao indicar que as pessoas não têm uma necessidade de autonomia inerente e de igual valor entre elas, há variações entre as pessoas. Assim, é preciso que se considere o aspecto individual e sua interação com o contexto do trabalho para uma explicação mais completa do fenômeno. Do ponto de vista prático, pode-se destacar duas possíveis aplicações. A primeira se refere aos processos de alocação de pessoal que deve considerar as preferências do indivíduo em relação ao WD. Assim, tanto processos seletivos quanto de realocação deveriam levantar informações a esse respeito e considerá-las. A segunda aplicação refere-se aos processos de redesenho do trabalho que visam ampliar a motivação e o engajamento para o trabalho. Nesse caso, o profissional precisa considerar 
as preferências dos trabalhadores para que os resultados desejados sejam alcançados. Não existem fórmulas que se apliquem a qualquer trabalhador, e a tarefa de gestão também passa por identificar essas preferências para garantir uma força de trabalho engajada.

Apesar das contribuições, cabe destacar que esses resultados foram obtidos a partir de uma pesquisa de corte transversal e do autorrelato. Seria interessante que estudos futuros testassem essa relação utilizando heteroavaliação das características do trabalho ou da variável dependente. Além disso, é importante ampliar esses estudos para todas as características do trabalho, já que há indícios de que esses fenômenos se comportam de forma diferenciada a depender da característica investigada. Com o avanço dos estudos sobre WD proporcionada pelo novo modelo proposto por Morgeson e Humphrey (2006), essa é uma agenda de pesquisa a ser perseguida.

\section{Referências}

Andersen, S. M., Chen, S., \& Carter, C. (2000). Commentaries on "The 'What' and 'Why' of Goal Pursuits: Human Needs and the Self-Determination of Behavior." Psychological Inquiry, 11(4), 269-318. doi:10.1207/S15327965PLI1104_02

Bakker, A. B., \& Demerouti, E. (2008). Towards a model of work engagement. Career Development International, 13(3), 209-223. doi:10.1108/13620430810870476

Bakker, A. B., Demerouti, E., \& Sanz-Vergel, A. I. (2014). Burnout and Work Engagement: The JD-R Approach. Annual Review of Organizational Psychology and Organizational Behavior, 1(1), 389-411. doi:10.1146/ annurev-orgpsych-031413-091235

Bakker, A. B., Demerouti, E., \& Xanthopoulou, D. (2012). How do engaged employees stay engaged? Ciencia \& Trabajo, 14((spec. issue)), 16-22. Recuperado de https://www.isonderhouden.nl/doc/pdf/arnoldbakker/articles/articles arnold_bakker_277.pdf

Borges-Andrade, J. E., Lemos Alves Peixoto, A., Queiroga, F., \& Pérez-Nebra, A. R. (2019). Adaptation of the Work Design Questionnaire to Brazil. Revista Psicologia: Organizações e Trabalho, 19(3), 720-731. doi:10.17652/ rpot/2019.3.16837

Brown, S. P. (1996). A meta-analysis and review of organizational research on job involvement. Psychological Bulletin, 120(2), 235-255. doi:10.1037/0033-2909.120.2.235

Buunk, B. P., \& Nauta, A. (2000). Why intraindividual needs are not enough: Human motivation is primarily social. Psychological Inquiry, 11(4), 279-283. Recuperado de https://www.jstor.org/ stable/1449621?seq=1\#page_scan_tab_contents

Carver, C. S., \& Scheier, M. F. (1999). Control theory: A useful conceptual framework for personality-social, clinical, and health psychology. Em R. F. Baumeister (Ed.), Key readings in social psychology: The self in social psychology (pp. 299-316). New York: Psychology Press.

Chen, C.-Y., Yen, C.-H., \& Tsai, F. C. (2014). Job crafting and job engagement: The mediating role of person-job fit. International Journal of Hospitality Management, 37, 21-28. doi:10.1016/j.ijhm.2013.10.006

Chinelato, R. S. de C., Ferreira, M. C., \& Valentini, F. (2015). Evidence of Validity of the Job Crafting Behaviors Scale. Paidéia (Ribeirão Preto), 25(62), 325-332. doi:10.1590/1982-43272562201506

Cordery, J., \& Parker, S. K. (2012). Work design: Creating jobs and roles that promote individual effectiveness. Em S. W. J. Kozlowski (Ed.), The Oxford handbook of organizational psychology (Vol. 1) (pp. 247-284). Oxford: Oxford University Press

De Jonge, J., \& Schaufeli, W. B. (1998). Job characteristics and employee well-being: a test of Warr's Vitamin Model in health care workers using structura equation modelling. Journal of Organizational Behavior, 19(4), 387-407. doi:10.1002/(SICI)1099-1379(199807)19:4<387::AID-JOB851>3.0.CO;2-9

Deci, E. L., \& Ryan, R. M. (2000). The "What" and "Why" of Goal Pursuits: Human Needs and the Self-Determination of Behavior. Psychological Inquiry, 11(4), 227-268. doi:10.1207/S15327965PLI1104_01

Dormann, C., \& Zapf, D. (2001). Job satisfaction: a meta-analysis of stabilities. Journal of Organizational Behavior, 22(5), 483-504. doi:10.1002/job.98

Edwards, J. R. (1991). Person-job fit: A conceptual integration, literature review, and methodological critique. Em C. L. Cooper \& I. T. Robertson (Eds.), International Review of Industrial and Organizational Psychology (Vol. 6) (pp. 283-357). Oxford: John Wiley \& Sons.

Fedor, D. B., Cladwell, S., \& Herold, D. M. (2006). The effects of organizational changes on employee commitment: A multilevel investigation. Personnel Psychology, 59(1), 1-29. doi:10.1111/j.1744-6570.2006.00852.x
Ferreira, M. C.; Mendonça, H. (2012). Saúde e Bem-estar no Trabalho: dimensões individuais e culturais. São Paulo: Casa do Psicólogo.

Ferreira, M. C., Valentini, F., Damásio, B. F., Mourão, L., Porto, J. B., Chinelato, R. S. de C., ... Pereira, M. M. (2016). Evidências adicionais de validade da UWES-9 em amostras brasileiras. Estudos de Psicologia, 21(4), 435-445. doi:10.5935/1678-4669.20160042

Gagné, M., \& Deci, E. L. (2005). Self-determination theory and work motivation. Journal of Organizational Behavior, 26(4), 331-362. doi:10.1002/job.322

Gagné, M., Senécal, C. B., \& Koestner, R. (1997). Proximal Job Characteristics, Feelings of Empowerment, and Intrinsic Motivation: A Multidimensional Model1. Journal of Applied Social Psychology, 27(14), 1222-1240. doi:10.1111/j.1559-1816.1997.tb01803.x

Greguras, G. J., \& Diefendorff, J. M. (2009). Different fits satisfy different needs: Linking person-environment fit to employee commitment and performance using self-determination theory. Journal of Applied Psychology, 94(2), 465-477. doi:10.1037/a0014068

Greguras, G. J., Diefendorff, J. M., Carpenter, J., \& Tröster, C. (2014). Personenvironment fit and self-determination theory. Em M. Gagné (Ed.), Oxford library of psychology. The Oxford handbook of work engagement, motivation, and self-determination theory (pp. 143-161). New York: Oxford University Press.

Hackman, J. R., \& Oldham, G. R. (1980). Work redesign. Reading, Mass.: AddisonWesley, 1980.

Harter, J. K., \& Blacksmith, N. (2013). Employee engagement and the psychology of joining, staying in, and leaving organizations. Em P. A. Linley, S. Harrington, \& N. Garcea (Eds.), Oxford Handbook of Positive Psychology and Work (pp. 121-130). New York: Oxford University Press.

Hu, L., \& Bentler, P. M. (1999). Cutoff criteria for fit indexes in covariance structure analysis: Conventional criteria versus new alternatives. Structural Equation Modeling: A Multidisciplinary Journal, 6(1), 1-55. doi:10.1080/10705519909540118

Humberg, S., Nestler, S., \& Back, M. D. (2018). Response Surface Analysis in Personality and Social Psychology. Social Psychological and Personality Science, 194855061875760. doi:10.1177/1948550618757600

Humphrey, S. E., Nahrgang, J. D., \& Morgeson, F. P. (2007). Integrating motivational, social, and contextual work design features: A meta-analytic summary and theoretical extension of the work design literature. Journal of Applied Psychology, 92(5), 1332-1356. doi:10.1037/0021-9010.92.5.1332

Kahn, W. A. (1990). Psychological Conditions of Personal Engagement and Disengagement at Work. Academy of Management Journal, 33(4), 692-724. doi:10.5465/256287

Kristof-Brown, A., Zimmerman, R., \& Johnson, E. (2005). Consequences of individuals' fit at work: A meta-analysis of person-job, person-organization, person-group, and personsupervisor fit. Personnel Psychology, 58(2), 281-342. doi:10.1111/j.1744-6570.2005.00672.x

Lauver, K. J., \& Kristof-Brown, A. (2001). Distinguishing between Employees' Perceptions of Person-Job and Person-Organization Fit. Journal of Vocational Behavior, 59(3), 454-470. doi:10.1006/jvbe.2001.1807

Leal, E. A., Miranda, G. J., \& Carmo, C. R. S. (2013). Teoria da autodeterminação: uma análise da motivação dos estudantes do curso de ciências contábeis. Revista Contabilidade \& Finanças, 24(62), 162-173. doi:10.1590/ S1519-70772013000200007

Lin, Y.-C., Yu, C., \& Yi, C.-C. (2014). The Effects of Positive Affect, Person-Job Fit, and Well-being on Job Performance. Social Behavior and Personality: An International Journal, 42(9), 1537-1547. doi:10.2224/sbp.2014.42.9.1537

Linley, P. A., Harrington, S., \& Garcea, N. (2013). Oxford handbook of positive psychology and work. New York, NY: Oxford University Press.

Lu, C., Wang, H., Lu, J., Du, D., \& Bakker, A. B. (2014). Does work engagement increase person-job fit? The role of job crafting and job insecurity. Journal of Vocational Behavior, 84(2), 142-152. doi:10.1016/j.jvb.2013.12.004

Meyer, J. P., \& Gagné, M. (2008). Employee Engagement From a Self-Determination Theory Perspective. Industrial and Organizational Psychology, 1(01), 60-62. doi:10.1111/j.1754-9434.2007.00010.x

Morgeson, F. P., \& Humphrey, S. E. (2006). The Work Design Questionnaire (WDQ): Developing and validating a comprehensive measure for assessing job design and the nature of work. Journal of Applied Psychology, 91(6), 1321-1339. doi:10.1037/0021-9010.91.6.1321

Parker, S. K., Morgeson, F. P., \& Johns, G. (2017). One hundred years of work design research: Looking back and looking forward. Journal of Applied Psychology, 102(3), 403-420. doi:10.1037/apl0000106

Petrou, P., Demerouti, E., Peeters, M. C. W., Schaufeli, W. B., \& Hetland, J. (2012). Crafting a job on a daily basis: Contextual correlates and the link to work engagement. Journal of Organizational Behavior, 33(8), 1120-1141. doi:10.1002/job.1783 
Pyszczynski, T., Greenberg, J. L., \& Solomon, S. (2000). Proximal and distal defense: A new perspective on unconscious motivation. Current Directions in Psychological Science, 9(5), 156-160. doi:10.1111/1467-8721.00083

Satorra, A., \& Bentler, P. M. (2001). A scaled difference chi-square test statistic for moment structure analysis. Psychometrika, 66(4), 507-514. doi:10.1007/ BF02296192

Schaufeli, W. B. (2012). Work Engagement: What Do We Know and Where Do We Go? Romanian Journal of Applied Psychology, 14(1), 3-10.

Schaufeli, W. B. (2013). What is engagement? Em C. Truss, K. Alfes, R. Delbridge, A. Shantz, \& E. Soane (Eds.), Employee Engagement in Theory and Practice. London: Routledge.

Schaufeli, W. B. (2017). Applying the Job Demands-Resources model. Organizational Dynamics, 46(2), 120-132. doi:10.1016/j.orgdyn.2017.04.008

Schaufeli, W. B., Bakker, A. B., \& Van Rhenen, W. (2009). How changes in job demands and resources predict burnout, work engagement, and sickness absenteeism. Journal of Organizational Behavior, 30(7), 893-917. doi:10.1002/ job.595

Schaufeli, W. B., Salanova, M., González-Romá, V., \& Bakker, A. B. (2002). The measurement of engagement and burnout: A two sample confirmatory factor analytic approach. Journal of Happiness Studies, 3, 71-92. doi:10.1023/A:1015630930326

Schneider, A., Hornung, S., Weigl, M., Glaser, J., \& Angerer, P. (2017). Does it matter in the long run? Longitudinal effects and interactions in the differentiated job demands-resources model. European Journal of Work and Organizationa Psychology, 26(5), 741-754. doi:10.1080/1359432X.2017.1347561

Schönbrodt, F. D., \& Humberg, S. (2018). RSA: An R pack-age for response surface analysis (version 0.9.12). doi:10.1080/1359432X.2017.1347561

Sekiguchi, T., \& Huber, V. L. (2011). The use of person-organization fit and person-job fit information in making selection decisions. Organizational Behavior and Human Decision Processes, 116(2), 203-216. doi:10.1016/j. obhdp.2011.04.001
Sender, G., \& Fleck, D. (2017). As Organizações e a Felicidade no Trabalho: Uma Perspectiva Integrada. Revista de Administração Contemporânea, 21(6), 764-787. doi:10.1590/1982-7849rac2017160284

Shuck, B., Reio, T. G., \& Rocco, T. S. (2011). Employee engagement: an examination of antecedent and outcome variables. Human Resource Development International, 14(4), 427-445. doi:10.1080/13678868.2011.601587

Stairs, M., \& Galpin, M. (2013). Positive Engagement: From Employee Engagement to Workplace Happiness. In P. A. Linley, S. Harrington, \& N. Garcea (Eds.), Oxford Handbook of Positive Psychology and Work (pp. 155-174). Oxford: Oxford University Press.

Sulistiowati, S., Komari, N., \& Dhamayanti, E. (2018). International Review of Management and Marketing Econjournals, 8(3), 75-80. Recuperado de https://ideas.repec.org/a/eco/journ3/2018-03-12.html

Tangirala, S., \& Ramanujam, R. (2008). Employee silence on critical work issues: The cross level effects of procedural justice climate. Personnel Psychology, 61(1), 37-68. doi:10.1111/j.1744-6570.2008.00105.x

Tims, M., Bakker, A. B., \& Derks, D. (2012). Development and validation of the job crafting scale. Journal of Vocational Behavior, 80(1), 173-186. doi:10.1016/j. jvb.2011.05.009

Truss, C., Alfes, K., Delbridge, R., Shantz, A., \& Soane, E. (2013). Employee Engagement in Theory and Practice. London: Routledge.

van Vianen, A. E. M. (2018). Person-Environment Fit: A Review of Its Basic Tenets. Annual Review of Organizational Psychology and Organizational Behavior, 5(1), 75-101. doi:10.1146/annurev-orgpsych-032117-104702

Vazquez, A. C. S., Magnan, E. dos S., Pacico, J. C., Hutz, C. S., \& Schaufeli, W. B. (2015). Adaptation and Validation of the Brazilian Version of the Utrecht Work Engagement Scale. Psico-USF, 20(2), 207-217. doi:10.1590/1413-82712015200202

Wrzesniewski, A., \& Dutton, J. E. (2001). Crafting a Job: Revisioning Employees as Active Crafters of Their Work. Academy of Management Review, 26(2), 179-201. doi:10.5465/amr.2001.4378011 